\title{
Le schisme anglais et l'essor du sentiment national
}

Jean-Pierre Moreau

\section{(2) OpenEdition \\ Journals}

Édition électronique

URL : http://journals.openedition.org/shakespeare/1272

DOI : 10.4000/shakespeare.1272

ISSN : 2271-6424

Éditeur

Société Française Shakespeare

Édition imprimée

Date de publication : 1 novembre 1994

Pagination : 67-95

Référence électronique

Jean-Pierre Moreau, "Le schisme anglais et l'essor du sentiment national 》, Actes des congrès de la Société française Shakespeare [En ligne], 12 | 1994, mis en ligne le 01 janvier 2007, consulté le 30 avril 2019. URL : http://journals.openedition.org/shakespeare/1272 ; DOI : 10.4000/shakespeare.1272 
COSMOPOLITISME ET INSULARITÉ 


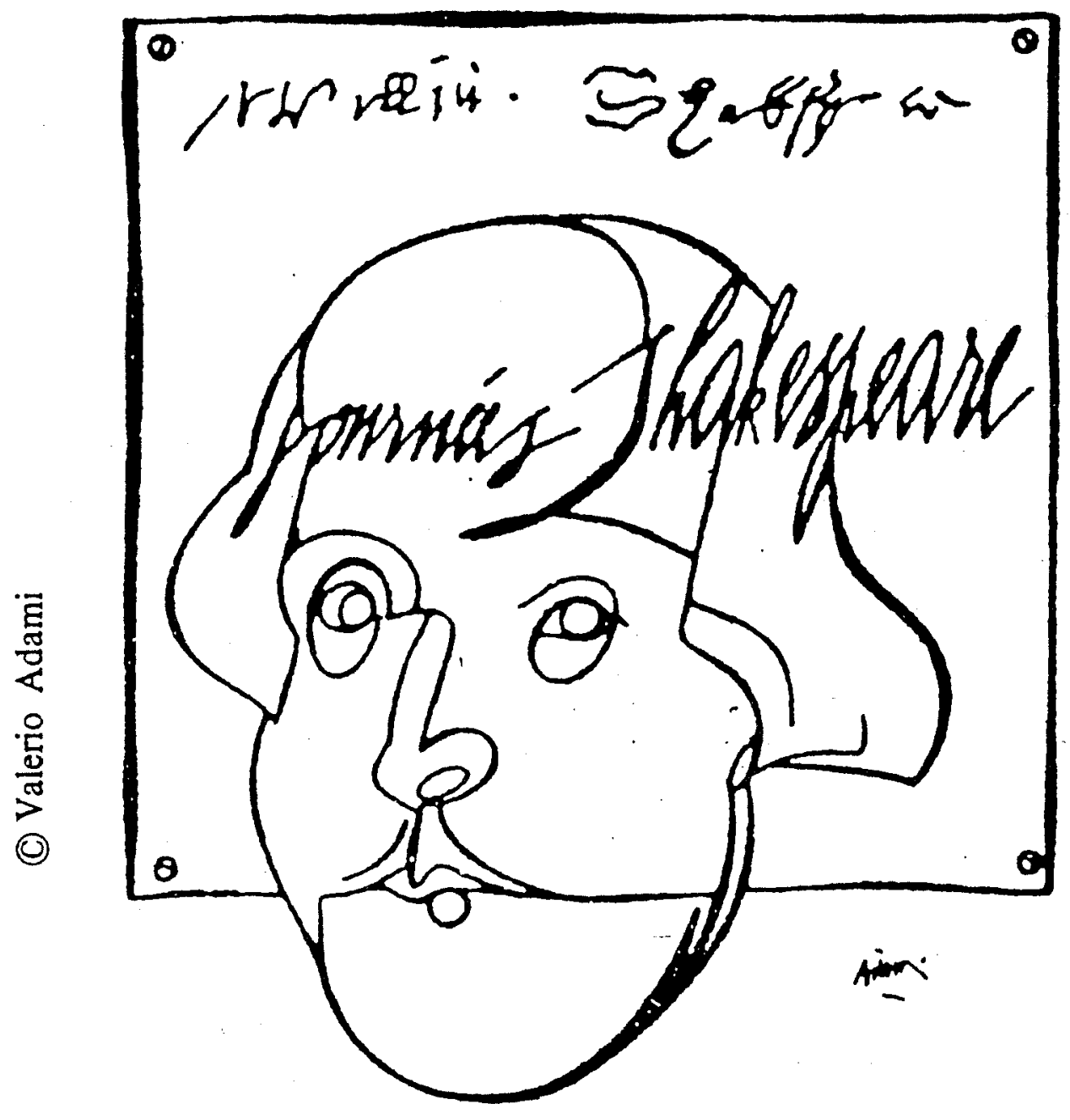

Affiche de Valerio Adami, spécialement conçue pour les journées Shakespeare 1979 


\section{SHAKESPEARE}

COSMOPOLITISME ET INSULARITÉ

Société Française Shakespeare

Actes du Congrès 1993

sous la direction

de

M. T. JONES-DAVIES

Ouvrage publié avec le soutien de

BARCLAYS

BARCLAYS BANK S.A

PARIS

LES BELLES LETTRES

1994 
Tous droits de traduction, de reproduction et d'adaptation réservés pour tous les pays.

(C) 1994 Société d'édition Les Belles Lettres, 95 bd Raspail 75006 Paris

ISBN 2.251.69122-7 


\section{Le schisme anglais et l'essor du sentiment national}

Les historiens font remonter la première affirmation du «nationalisme» à la Révolution française (parfois un peu plus tôt en Angleterre) lorsque la nation tout entière revendique le pouvoir politique. Nous parlerons donc ici de «sentiment national» qui se manifeste de mille façons à l'époque de Shakespeare. Il apparaît, notamment, dans des textes littéraires bien connus qui chantent les louanges de l'Angleterre en termes emphatiques.

John Lyly écrit en 1580 :

O thrise happy England where such Counsaylours are, where such people liue, where such vertue springeth ${ }^{1}$.

Dans les années 1590, Spenser retrace, dans The Faerie Queene, l'histoire légendaire des rois saxons et fait dire à l'un d'eux :

Deare countrey, $\mathrm{O}$ how dearely deare

Ought thy remembraunce, and perpetuall band

Be to thy foster Childe, that from thy hand

Did commun breath and nouriture receaue?

How brutish is it not to vnderstand,

How much to her we owe, that all vs gaue,

That gaue vnto vs all, what euer good we haue.

(Livre 2, chant 10, strophe 69)

Au même moment, Shakespeare donne à ce sentiment son expression la plus noble et la plus achevée dans le discours de John of Gaunt (Richard II) qui célèbre la grandeur (passée) de son pays (et répète le mot «dear» plus souvent encore que Spenser) :

This royal throne of kings, this scepter'd isle,

This earth of majesty, this seat of Mars,

This other Eden, demi-paradise,

This fortress built by Nature for herself

Against infection and the hand of war,

This happy breed of men, this little world,

This precious stone set in the silver sea,

Which serves it in the office of a wall, 
Or as a moat defensive to a house,

Against the envy of less happier lands,

This blessed plot, this earth, this realm, this England,

This nurse, this teeming womb of royal kings,

Fear'd by their breed and famous by their birth,

Renowned for their deeds as far from home, -

For Christian service and true chivalry, -

As is the sepulchre in stubborn Jewry

Of the world's ransom, blessed Mary's Son:

This land of such dear souls, this dear, dear land,

Dear for her reputation through the world,

(Acte Il, scène 1, vers 40-58)

Mais la fierté nationale prend des formes moins sublimes qui n'en montrent que mieux à quel point elle est répandue. Dans Description of Engiand (1578), William Harrison affirme que «les Anglais sont supérieurs aux habitants des pays plus chauds», que «la beauté des Anglaises dépasse d'ordinaire celle des plus belles femmes du continent» et en particulier qu'après quarante ans, elles sont plus agréables à regarder «que les femmes de France et de divers autres pays» ${ }^{2}$.

On peut se demander quand s'est développée cette conscience nationale car les opinions divergent sur ce point. Selon John Guy, «national identity developed late and was the result, rather than the cause, of the Protestant Reformation ${ }^{3}$. Ce qui tendrait à dénoncer un anachronisme dans Richard II, le duc prononçant sa tirade en 1399. Pour J.W. Allen, en revanche, «[...] in England in 1529 there already existed clear outlines of a national State, and along with this a national sentiment and desire for complete independence ${ }^{4}$. Ces deux auteurs mettent en cause linfluence des transformations religieuses sur le sentiment national (1529, chez Allen, marque le début du «Reformation Parliament»), mais outre leur désaccord, ils procèdent par affirmations et n'examinent pas en détail la question centrale : dans quelle mesure le schisme est-il à l'origine de ce sentiment ou en constitue-t-il seulement le résultat?

En fait, nous verrons que Shakespeare n'avait pas tort : un indéniable patriotisme existait déjà à l'époque de Richard Il. Il allait s'accentuer par la suite et permettre une acceptation relativement aisée de la rupture avec Rome dans les années 1530 - il faut rappeler que cette rupture provient du désir de donner un héritier mâle au pays et d'éviter ainsi ce qui aurait pu être une catastrophe nationale ${ }^{5}$. Finalement, la situation créée par le schisme 
joue, à son tour, un rôle en modifiant le sentiment national, en lui donnant une intensité et une qualité nouvelles. Le schisme ne génère pas ce sentiment, il l'utilise puis le transforme au point d'engendrer à la fin du XVI $\mathrm{XV}^{e}$ siècle et au XVII ${ }^{e}$ siècle des conceptions politiques et intellectuelles qui ébranlent le cadre traditionnel de la pensée élisabéthaine.

Le Moyen Age voit s'affirmer deux formes parallèles de patriotisme. L'une s'organise autour du souverain et revêt souvent un caractère religieux. L'autre, plus tardive, tend au contraire à se dégager de la personne du roi et, selon les circonstances, concerne une fraction variable de la nation; il lui arrive alors de mettre l'accent sur des composantes «païennes» comme la race, le territoire, la langue, voire la culture ou l'économie. Jusqu'au XVI ${ }^{e}$ siècle, les deux formes - monarchique et nationale - demeurent en général séparées mais ne cessent de poser des problèmes à l'Église. Tantôt celle-ci les encourage (sacralisation du pouvoir royal, fusion des Normands et des Saxons en un seul peuple de chrétiens) mais au risque de voir le monarque ou un secteur de l'opinion critiquer le clergé lorsqu'ils prennent conscience de leur force. Tantôt elle les freine (intervention contre Jean sans Terre) quitte à payer, plus tard, le prix de la revanche.

Le «patriotisme monarchique» prévaut lorsque les rois d'Angleterre veulent égaler puis dépasser les autres souverains d'Europe. Les anciens ducs de Normandie cherchent à se hisser au même niveau d'autorité et de respectabilité que les rois de France en particulier. Imitation, rivalités, emprunts (de titres) alimentent cette forme de sentiment national.

L'exemple le plus frappant est celui des pouvoirs thaumaturgiques qui révèlent le caractère mixte - sacrê et profane - de la personne du roi. Dès le règne de Henry $\mathrm{I}^{\mathrm{er}}(1100-1135)$, ce dernier est censé guérir les écrouelles comme les Capétiens. Sous Edward II (1307-1327) on ajoute l'épilepsie pour faire bonne mesure.

Le même Edward II tente, sans succès, d'obtenir de Rome que soient reconnues les propriétés d'une sainte ampoule, visiblement calquée sur celle héritée en France de Clovis, et qui aurait été remise à Thomas Becket par la Vierge. Henry IV (1399-1413) adoptera cette nouvelle onction et n'aura ainsi plus rien à envier 
aux Valois dans ce domaine ${ }^{6}$ : la monarchie anglaise jouit du même caractère sacré et de la même protection divine que son homologue française.

Il manque cependant un titre officiel qui proclame les mérites religieux du roi d'Angleterre. Henry VIII n'aura de cesse d'être à parité avec son beau-père, Sa Majesté «Très Catholique», et avec son «cousin» François Ier, le roi «Très Chrétien». La récompense vient en 1521 lorsqu'il est nommé «Défenseur de la Foi» par Léon $X$ à la suite de son livre contre Luther (après que «Protecteur du Saint-Siège» et «Défenseur de l'Église» aient été envisagés).

L'émulation s'exprime aussi dans l'ordre temporel. Très tôt les rois de France avaient revendiqué auprès de Rome une indépendance totale vis-à-vis de l'Empire. Dès 1202, la bulle Per venerabilem (Innocent III) avait fait d'eux des «empereurs en leur royaume» ${ }^{7}$, position confirmée en 1311 par Rex gloriae adressée à Philippe IV le Bel. En 1397, le chancelier anglais dit de Richard II qu'il est «(le) roy entier emperour de son roialme» ${ }^{8}$. Les rois d'Angleterre n'ont pas trop à craindre les immixtions de l'empereur. On ne relève qu'un seul exemple de précaution prise en ce domaine : en 1416, l'empereur Sigismond n'aurait été autorisé à débarquer à Douvres qu'après avoir promis de ne revendiquer aucune autorité en Angleterre ${ }^{9}$. Il n'en va pas de même pour les relations avec la France ou avec Rome.

Les guerres jouent un rôle essentiel aussi bien dans l'affirmation de la forme «monarchique» du patriotisme que dans l'essor de sa forme «nationale». Commencée comme une querelle féodale, la guerre de Cent Ans devient une lutte dynastique et finalement un conflit entre deux nations au cours duquel les rois d'Angleterre s'approprient le titre de roi de France. Chaque victoire anglaise (Crécy, 1346, Poitiers, 1356 et surtout Azincourt, 1415) est interprétée comme un signe de préférence divine à l'égard du souverain. Une chronique écrite deux ans après Azincourt, Gestu Henri Quintu, présente le souverain comme verus electus dei. Mais en même temps, imitant là-aussi ce qui avait été fait en France, certains responsables anglais comparent leur pays à Isrä̈ ${ }^{10}$. Dieu a choisi entre deux peuples car deux royaumes «qui n'ont pas de supérieur» sur terre ne peuvent être départagés que par le créateur luimême. Ainsi glisse-t-on de la première à la deuxième forme de patriotisme. Des rois facilitent cette évolution. Henry V (1413-1422) insiste sur le fait qu'il n'est que l'instrument de Dieu qui a voulu la 
victoire d'Azincourt pour l'Angleterre. En plusieurs circonstances certains n'hésitent pas à attribuer la nationalité anglaise au créateur. Un évêque de Rochester craint qu'en raison des péchés britanniques, Dieu ne retire sa protection: «God who was accustomed to being English will abandon us» ${ }^{11}$.

Les mouvements d'humeur vis-à-vis de la papauté apparaissent aussi aux deux niveaux. Il ne s'agit jamais de rompre avec Rome (comme le fait Louis XII en 1511) ou même de revendiquer une autonomie aussi large que celle obtenue par la France avec la pragmatique sanction de Bourges en 1438. Mais Henry VIII - qui avait offert ses services au pape pour combattre Louis XII s'offusque en 1515 de la témérité du clergé et d'une décrétale de Léon $\mathrm{X}$ qui contredit la loi anglaise sur le bénéfice de clergie :

We are, by the sufferance of God, King of England and the Kings of England in times past never had any superior but God ${ }^{12}$.

Un sentiment de méfiance, voire d'hostilité, anime parfois certaines fractions de la population anglaise. Les taxes pontificales irritent, surtout celles qui suivent la déclaration de vassalité de Jean sans Terre après l'humiliation de l'interdit et de l'excommunication prononcés par Innocent III (1208-1213). D'autant que cet argent est utilisé à des fins jugées temporelles par de nombreux Anglais. Lorsque la papauté s'installe à Avignon (1309-1378), deux antipathies se confondent: les papes sont trop aux ordres de l'ennemi français. Dans la deuxième moitié du XIV ${ }^{e}$ siècle une série de statuts - «Statute of Provisors» et «Statutes of Praemunire» - limite l'influence de Rome en Angleterre notamment en punissant lintroduction de certaines bulles pontificales et en interdisant de recourir à une justice étrangère. Le parlement et les couches sociales qu'il représente (propriétaires terriens surtout) s'unissent au roi pour imposer une plus grande indépendance nationale.

Cette tendance prend une dimension populaire avec la contestation radicale de l'autorité du Saint-Siège - et des ecclésiastiques - que proposent Wycliffe et ses partisans ultérieurs les Lollards. La priorité absolue accordée à la Bible permet de nier le rôle d'intermédiaire que le clergé remplit traditionnellement entre Dieu et les fidèles. C'est au roi que Wycliffe demande de réorganiser la vie religieuse, de réformer l'Église d'Angleterre et de rejeter la suprématie de Rome. Le mouvement cesse de se développer après l'échec de la «Révolte des paysans» (1381) quand tout sou- 
tien aristocratique lui est retiré mais il survit au début du XVI ${ }^{e}$ siècle, surtout parmi les marchands et les artisans.

Au-delà des éléments politico-religieux, il est aussi une composante culturelle du sentiment national. A côté du latin utilisé par les clercs et du français que conservent les juristes, l'anglais qui n'est devenu langue maternelle des rois d'Angleterre qu'au XIII ${ }^{\mathrm{e}}$ siècle, gagne du terrain. Langland et surtout Chaucer en font la langue littéraire nationale. C'est en anglais que Wycliffe dénonce l'autorité de Rome et commence la traduction de la Bible. En 1346, lorsque Edward III s'efforce de galvaniser les énergies contre le roi de France, il affirme que ce dernier veut supprimer la langue anglaise («root out the English tongue») ${ }^{13}$. La défense de la langue s'identifie à la défense du pays. Lors du concile de Constance (1414-1418), les ecclésiastiques anglais revendiquent, contre leurs homologues français, un vote par nations en ces termes :

Whether nation be understood as a people marked off from others by blood-relationship and habit of unity or by peculiarities of language (the most sure and positive sign and essence of a nation in divine and human law) [...] or whether nation be understood, as it should be, as a territory equal to that of the French nation, England is a real nation ${ }^{14}$.

Ainsi se définit la nation par la langue, le territoire, les coutumes et les liens du sang - et par opposition à la France.

Le développement commercial facilite, lui aussi, une prise de conscience des intérêts britanniques. Les premiers linéaments du mercantilisme que l'on a pu définir comme «un système de nationalisme économique» ${ }^{15}$ se dessinent dès la fin du XIVe siècle. Le contrôle des activités des marchands continentaux (vénitiens et hanséates surtout) s'accompagne d'avantages substantiels accordés aux fabricants et négociants locaux. En 1380, un statut réserve le transport des marchandises aux navires anglais ${ }^{16}$. Entre 1485 et 1490 , Henry VII fait voter des lois qui confirment ces privilèges et qui, bien avant les «Navigation Acts» de 1651 et 1660, exigent que les équipages soient britanniques ${ }^{17}$. Il s'agit de renforcer une flotte marchande capable de jouer un rôle dans la défense du pays en cas de guerre. Cette évolution exacerbe l'orgueil national qui prend parfois des formes détestables comme lors des émeutes dirigées contre les commerçants étrangers de Londres en mai 1517 (Evil May Day). La fierté des Anglais agace les visiteurs étrangers. 
Un Italien les décrit ainsi en $1500:[\ldots]$ the-English are great lovers of themselves, and of everything belonging to them ; they think that there are no other men than themselves, and no other world but England ${ }^{18}$.

Sans doute, à ne citer que des preuves de l'existence du sentimental national est-on conduit à en exagérer l'importance. Les divisions sociales et géographiques de l'Angleterre ${ }^{19}$, le manque de moyens de communication empêchent qu'il se développe au-delà des centres urbains et des groupes les plus instruits. Les liens féodaux restent très forts dans le Nord du pays et, comme le montre la révolte de 1536-37 connue sous le nom de Pèlerinage de la Grâce, l'allégeance au seigneur local et à Rome l'emporte nettement sur le respect pour le nouveau maillon intermédiaire que constitue le pays. Il n'en reste pas moins que tous les exemples donnés révèlent les débuts d'une prise de conscience indubitable de l'unité et de l'identité nationales. Diverses formes de subjection ou d'entraves sont de plus en plus mal ressenties aussi bien par le souverain que par les couches les plus actives de la population. Plus important encore, les souhaits du roi peuvent s'identifier avec ceux de cette population.

\section{II}

C'est ce que va réussir Henry VIII au début des années 1530 lorsqu'il utilise l'anticléricalisme de plusieurs groupes sociaux dans sa controverse avec le Saint-Siège. Puisque le clergé dépend du pape, l'insoumission du roi à Rome et l'hostilité de certains laïques envers les prélats se confondent avec, comme dénominateur commun, le sentiment national. Ce sentiment sert à justifier aussi bien les manœuvres dirigées contre Clément VIl que la rupture de 1534 qui scelle la nationalisation de l'Eglise et l'avènement d'un Etat-nation totalement indépendant. On observe deux sortes de recours au sentiment national pour prouver la validité de la politique adoptée en Angleterre. L'une, négative, consiste à dénoncer les erreurs ou les abus de pouvoir de Rome, l'autre, qui se veut plus constructive, recherche les «véritables traditions nationales».

La loi de 1536 (28 Henry VIII, c. 10) qui résume toute la propagande et toutes les mesures prises entre 1532 et 1534 condamne avec virvlence «the infinite abominations and mischiefs» de «l'évêque de Rome» ${ }^{20}$ — des termes très généraux qui 
recouvrent des griefs précis dont une liste est fournie dans le même texte. Celui «que certains appellent le pape»:

[..] did obfuscate and wrest God's holy word and testament a long season from the spiritual and true meaning thereof to his worldly and carnal affections, as pomp, glory, avarice, ambition and tyranny, covering and shadowing the same with his human and politic devices, traditions and inventions, set forth to promote and stablish his only dominion, both upon the souls and also the bodies and goods of all Christian people, excluding Christ out of his kingdom and rule of man his soul as much as he may, and all other temporal kings and princes out of their dominions which they ought to have by God's law upon the bodies and goods of their subjects ${ }^{21}$.

La «tyrannie de Rome», le «pouvoir usurpé» inclut au plan matériel, le versement du revenu annuel d'un diocèse lors de la nomination de chaque nouveau prélat. Les deux lois sur les annates (1532 et 1534) limitent puis interdisent désormais cette pratique ${ }^{22}$. Lorsque le mercantilisme vise à accumuler la plus grande quantité possible de métaux précieux, de nombreux Anglais s'offusquent que «de larges et inestimables sommes d'argent sortent chaque jour du royaume et l'appauvrissent» comme le répètent les deux lois ${ }^{23}$. Un troisième statut reprend les mêmes expressions pour abolir le paiement de toute indemnité ou impôt à Rome et, en particulier, ce que l'on appelait «Peter's Pence», versé par les propriétaires fonciers importants ${ }^{24}$. Les sommes concernées par le «denier de saint Pierre» ne sont pas très conséquentes (deux cents livres pour l'ensemble du pays) mais elles sont déboursées par des laïques qui partagent avec le roi ce que l'on pourrait appeler le même ressentiment national. (Le statut est rédigé sous forme de pétition adressée par les Communes au souverain).

Une controverse avait opposé Tyndale (Obedience of a Christian Man, 1528) et Thomas More (Supplication of Souls, 1529) sur l'origine du «denier de saint Pierre»25. Bien que 'Thomas More ait eu raison de dire que l'impôt était bien antérieur au règne de Jean sans Terre, Tyndale visait un endroit sensible lorsqu'il affirmait que «Peter's pence» avait été la conséquence de l'interdit prononcé par Rome au début du XIII ${ }^{e}$ siècle. Les discussions se poursuivront tout au long du règne de Henry VIII et de ses trois enfants sur la nature de la soumission de Jean sans Terre à Innocent III. Richard Grafton répète le point de vue henricien dans sa Chronicle de $1569^{26}$ : Jean avait été injustement contraint à se 
déclarer le vassal du pape au grand détriment de la couronne et du royaume.

Mais les «abus de pouvoir» pontificaux n'appartiennent pas qu'au passé médiéval. La question du «divorce» révèle, selon Henry VIII et ses partisans (surout le clan des Boleyn) une double usurpation d'autorité de la part de Rome : temporelle et spirituelle.

La loi relative au denier de saint Pierre dénonce un prétendu pouvoir du pape de dispenser les Anglais d'obéir aux lois de leur pays :

[...] his abusing and beguiling your subjetcs, pretending and persuading to them that he hath full power to dispense with all human laws, uses and customs of all realms in all causes which be called spiritual, which matter hath been usurped and practised by him and his predecessors by many years in great derogation of your imperial crown and authority royal, contrary to right and conscience ${ }^{27}$.

Il est fait allusion ici à ce qui a probablement le plus choqué Henry VIII dans ses démêlés avec Clément VII : la citation à comparaître à Rome devant le tribunal de la Rote (1530) - une insulte personnelle et à l'ensemble du pays. Aucun Anglais, dit-il, ne peut-être jugé hors d'Angleterre : ne extra Angliam litigare cogantur ${ }^{28}$. Il demande que le procès soit confié, malgré l'appel de Catherine d'Aragon (1530), soit à trois évêques anglais, soit à l'ensemble du clergé de la province de Canterbury, soit au seul primat d'Angleterre.

Un autre statut de 1534 s'en prend aux immixtions pontificales dans les questions de succession à la tête d'un pays : «the bishop of Rome [...] hath presumed in times past to invest who should please them to inherit in other men's Kingdoms and dominions, which thing we your most humble subjects both spiritual and temporal do most abhor and detest ${ }^{29}$. Une nouvelle fois, les élites politiques épousent la querelle du roi et font correspondre leur patriotisme avec les intérêts du souverain. Le même texte permet de dénoncer, au nom de l'unité et de la tranquillité du royaume, tous les «princes étrangers» qui apportent leur soutien à des prétendants illégitimes ${ }^{30}$.

Pour en revenir aux papes, leur ingérence dans les affaires intérieures d'un royaume constitue plus qu'un manquement aux règles de la politique, c'est une infraction à la loi divine qu'ils sont chargés de faire respecter. "Contrary to the great and inviolable grants of jurisdictions given by God», «did obfuscate and wrest 
God's holy word and testament» répètent les préambules des statuts $^{31}$. Cette idée avait été à l'origine de la demande d'annulation du mariage avec Catherine d'Aragon. En effet, la dispense pontificale de 1504 ayant permis à Henry d'épouser la veuve de son frère Arthur est jugée contraire à la loi de Dieu telle qu'elle apparaît dans la Bible (Lévitique 18, 16 et 20, 21).

Petit à petit, les Anglais en viennent à penser que la papauté a usurpé non seulement des pouvoirs temporels mais son autorité spirituelle qui ne devrait pas s'étendre, dans le meilleur des cas, au-delà du diocèse de Rome ${ }^{32}$. L'Angleterre est donc fondée à revendiquer son indépendance religieuse et elle le fait avec le sentiment de rester fidèle au véritable catholicisme. Selon une loi de 1534, déjà citée, elle ne s'écarte pas «from the congregation of Christ's Church in any things concerning the very articles of the Catholic Faith or Christendom» ${ }^{33}$. Comme par temps de brouillard, c'est le continent qui est isolé ; le nationalisme météorologique a de solides antécédents.

Hugh Latimer, le prédicateur et évêque henricien, résume les griefs patriotiques, politiques et théologiques que l'on fait à Rome en une formule : "That Italian bishop, yonder, the Devil's chaplain» ${ }^{34}$. Le désir d'indépendance nationale implique que les Anglais n'aient qu'un seul maître. En mai 1532, Henry VIII prétend découvrir que les prélats ne sont qu'à moitié ses sujets - et encore - puisqu'il prètent au pape un serment incompatible avec l'obéissance due au souverain :

All the prelates at their consecration make an othe to the pope clene contrary to the othe that they make to us, so that they seme to be his subjectes and not ours ${ }^{35}$.

En fait, dès décembre 1530 , il avait établi une différence entre «entier Englishmen» et «Englishmen papisticate» ${ }^{36}$.

C'est bien le sentiment de nationalité anglaise et d'identité nationale qui conduit progressivement au schisme. Sans que rien n'ait jamais été programmé ou inéluctable ${ }^{37}$, l'entente entre le parlement et le roi a permis de priver le pape de tous ses revenus anglais, puis de son autorité juridictionnelle, disciplinaire et finalement doctrinale en Angleterre.

Les justifications de ce bouleversement apparaissent à partir de 1530 sous forme de défense des traditions britanniques ou, comme on disait alors, des «privilèges du royaume». Henry VIII en- 
courage un mouvement d'origine humaniste qui recherche les vestiges du passé et l'utilise à ses propres fins. Il envoie des émissaires dans les bibliothèques d'Angleterre et d'Europe - y compris au Vatican - afin de justifier sa position dans l'affaire du «divorce» et, en particulier, afin de prouver qu'un Anglais ne saurait comparaître devant une juridiction étrangère. Il place donc la discussion sur le plan de l'histoire après avoir exploré celui du droit (canon) et de l'exégèse biblique.

Un ensemble de documents connus sous le nom de Collectanea satis copiosa ${ }^{38}$ lui est remis en septembre 1530 par Edward Foxe et Cranmer. A partir de ces textes d'origine fort diverse - pères de l'Église, décisions de conciles, théologiens ou canonistes médiévaux et, surtout, des chroniqueurs anglais comme Bede, William of Malmesbury ou Geoffrey of Monmouth s'élabore une conception politique tout à fait nouvelle que le gouvermement juge authentique et vénérable.

On peut distinguer trois aspects qui tous flattent le sentiment national. Le premier concerne l'étendue du pouvoir royal, assimilé à celui d'un empereur, et met l'accent sur la formule Rex in regno suo est imperator. Le Conseil du roi a dû en discuter puisque trois membres influents de cette assemblée, les ducs de Norfolk et de Suffolk, et le comte de Wiltshire (père d'Anne Boleyn) l'utilisent au cours des mois qui suivent ${ }^{39}$. Si le mot «empire» avait déjà été employé, c'était très exceptionnellement ${ }^{40}$ ou pour indiquer que le roi était le souverain de deux pays (Angleterre et France par exemple). Maintenant il signifie que Henry VIII n'a pas de supérieur sur terre, que le clergé doit lui être soumis : c'est au début de 1531 qu'il revendique le titre de «chef suprême de l'Église d'Angleterre» et même la charge des âmes de ses sujets. A l'appui de cette thèse audacieuse Collectanea se réfère aux Leges Anglorum selon lesquelles le premier roi chrétien d'Angleterre, Lucius ${ }^{\text {Ier }}$, à la fin du $I I^{e}$ siècle, aurait accordé à l'Église d'Angleterre ses franchises et ses possessions. Le pape de l'époque, Éleuthère (175-189), lui aurait envoyé une lettre, incluse dans Collectanea, lui reconnaissant un pouvoir spirituel autant que temporel et utilisant la formule «vicaire de Dieu» (vicarius del) à son égard ${ }^{41}$.

Le deuxième élément est la conséquence immédiate de ce qui précède : la totale indépendance juridictionnelle de l'Angleterre vis-à-vis de Rome. Les procès doivent être jugés sur place, sans appel possible au tribunal de la Rote. Selon Collectanea, des conciles confirment ce fait : Nicée (325), Carthage (419), Constance 
(1414-1418), etc., de même que la donation de Constantin. Celleci prouve que l'origine du pouvoir temporel des papes est humaine, et non divine, et que les rois ou les empereurs peuvent reprendre ce qui n'a été que «prêté» à Rome par leurs prédécesseurs (Constantin ou Lucius par exemple) ${ }^{42}$.

Enfin, troisième élément, une généalogie prestigieuse rehausse encore la gloire de la monarchie anglaise. Les chroniques montrent que les rois d'Angleterre comptent non seulement le légendaire Arthur parmi leurs devanciers mais aussi Brutus, arrière petit-fils d'Enée, et l'empereur Constantin lui-même. La mère de ce dernier aurait été anglaise et Constantin en personne aurait régné en Angleterre ${ }^{43}$ ce qui justifierait une interprétation littérale de la formule «Rex est imperator». Au nom de cette ascendance, Henry VIII souhaite recouvrer l'héritage normal des souverains anglais que la négligence de certains et les manoeuvres des papes ont fait tomber en désuétude.

Cette nouvelle théorie du pouvoir monarchique échappe bientôt au caractère confidentiel du manuscrit et des conversations privées; elle éclate, en 1533, dans la célèbre loi sur les appels dont le préambule sonne comme une fanfare :

Where by divers sundry old authentic histories and chronicles it is manifestly declared and expressed that this realm of England is an empire, and so hath been accepted in the world, governed by one supreme head and king having the dignity and royal estate of the imperial crown of the same $\mathrm{e}^{44}$.

Bien au-delà de son utilité immédiate - rendre caduc l'appel de Catherine à Rome - ce texte pose comme axiome de base la souveraineté de l'Angleterre et insiste sur le fondement divin du pouvoir royal («by the goodness and sufferance of Almighty God») sans aucun intermédiaire sur terre («next to God»). Toute autorité dérive de celle du roi - conception «descendante» du pouvoir - y compris celle du clergé placé sous le contrôle exclusif du monarque. Cette souveraineté est essentiellement territoriale : personne ne peut intervenir de l'extérieur et nul, dans le pays, ne peut échapper aux lois du royaume.

Inutile de dire que les justifications laissent beaucoup à désirer. Les «divers sundry old authentic histories and chronicles» restent dans le vague; aucune n'est citée car le gouvernement sait à quel point les «preuves» sont fragiles. Il renonce à une allusion explicite à la lettre du pape Eleuthère (prévue dans un avant-pro- 
jet) ${ }^{45}$ car c'est un faux qui date du XIII ${ }^{e}$ siècle. Utiliser la donation de Constantin ne peut tromper que ceux qui ignorent la démonstration de Lorenzo Valla vieille de près d'un siècle. La prétendue christianisation de l'Angleterre au tout début de notre ère, le règne anglais de Constantin et bien d'autres légendes relatives au roi Arthur et même au séjour du Christ outre-Manche ne sauraient faire illusion à un moment où les historiens deviennent plus exigeants. Polydore Virgile (ou Vergilio) qui écrit une Historia anglicana à la demande de Henry VIII (première édition, 1534), conteste certains de ces «faits».

La raison s'accommode mal du flou historique et des altérations de la vérité mais le but du roi est de faire appel aux sentiments de ses sujets : méfiance, voire hostilité envers Rome, fierté à l'égard du passé du pays et du rayonnement de la couronne. Il importe peu que les couleurs ne soient pas authentiques, l'essentiel est qu'elles plaisent. Et elles ne pouvaient plaire qu'en jouant sur la corde déjà sensible du sentiment national.

\section{III}

Les modifications politico-religieuses des années 1530 ont aussi contribué, ensuite, à transformer ce sentiment. L'isolement de l'Angleterre, les nouvelles structures constitutionnelles, les difficultés rencontrées pour les mettre en application, les luttes de factions pour les orienter dans un sens réformiste ou conservateur ont profondément influé sur les façons de penser et donc sur l'un des piliers qui soutenaient l'édifice : la conscience que les Anglais avaient de leur identité et de leur originalité a été nettement renforcée et cette conscience a pris une tournure originale qui tranche encore aujourd'hui dans le concert des nations.

On peut distinguer quatre types de conséquences du schisme sur le sentiment national : la défense obstinée d'une totale indépendance à l'égard de l'étranger (alors que cette indépendance était partielle avant 1534$)^{46}$, l'insistance sur l'unité de la société anglaise et donc de la nation, le devoir d'obéissance unanime au souverain et, surtout, la dimension religieuse que prend la conscience nationale.

L'indépendance vis-à-vis de l'étranger signifie, avant tout, l'indépendance envers Rome revendiquée dans toutes les œuvres de 
propagande henricienne, édouardienne et élisabéthaine. Depuis $D e$ Vera Differentia Regiae Potestatis et Ecclesiasticae de Edward Foxe (1534) jusqu'au traité de John Bridges, un demi- siècle plus tard, A Defense of the Government Established (1584), chacun insiste sur le fait que Dieu n'a pas confié le gouvernement de l'Eglise aux «évêques de Rome»; les Écritures («Tu es Pierre ...» et «Pais mes brebis») n'attribuent, au mieux, qu'une primauté d'honneur à saint Pierre et à ses successeurs. Il n'aurait donc jamais dû y avoir de centralisation de ce gouvernement et de chef unique pour toute la chrétienté.

Concrètement l'indépendance à l'égard de Rome se marque par les lois de 1532-1536 citées plus haut mais aussi par la dissolution des monastères (1536-1540), entre autres exemples. On avance des raisons morales (vices) ou théologiques (association avec le dogme du purgatoire), il en est aussi de «nationalistes»: les monastères s'inscrivent souvent dans de vastes réseaux européens qui échappent au contrôle du gouvernement anglais ${ }^{47}$.

Rome inquiète aussi par les menaces d'invasion que pourrait susciter le pape en vertu des conceptions médiévales d'intervention militaire dans les pays frappés d'interdit ${ }^{48}$. En décembre 1538 , Paul III se prépare à promulguer la bulle d'excommunication contre Henry VIII et certains Anglais, dont le cardinal Pole, s'activent pour organiser une croisade contre $1^{1}$ Angleterre ${ }^{49}$. L'entreprise n'aura pas lieu mais une atmosphère de crise règne à londres au début de 1539. le pays organise ses milices, renforce ses défenses; Henry visite les ports de la côte sud, passe des troupes en revue dans la capitale. Des rumeurs courent de flottes étrangères rassemblées à Anvers et à Boulogne. La peur partagée fortifie la cohésion nationale qu'encourage l'évêque de Durham en ces termes :

[...]Thou Englishman, take courage into thee and be nothing afraid. Thou hast God on thy side who hath given this realm to the generation of Englishmen, to every man his degree, after the laws of the same; thou hast a noble, victorious and virtuous King, hardy as a lion, who will not suffer thee to be so devoured by such wild beasts. Only take an English heart into thee and mistrust not God ${ }^{50}$.

La situation paraîtra moins critique après la bulle Regnans in excelsis lancée par Pie V en 1570 pour excommunier et déposer Elizabeth. Le soutien international («le glaive temporel») fait défaut au pape et les forces catholiques ont beaucoup décliné en Angleterre. Cependant l'échec même de cette tentative renforce le patriotisme anglais et les autres déconvenues des adversaires d'Eli- 
zabeth (Rébellion du Nord en 1569, complots Ridolfi et Babington, «Invincible Armada» et alertes de 1593 puis 15961597) accroissent l'assurance du pays qui communie dans le culte de la souveraine comme il ne l'avait jamais fait auparavant ${ }^{51}$. Les pamphlets politiques, les sermons manifestent un patriotisme extraordinaire.

Le danger donne corps à l'idée exprimée par Machiavel dans Libro dell'Arte della Guerra (1521) selon laquelle le pays tout entier doit être prêt à se lever en armes contre l'agresseur plutôt que de faire appel à des corps de mercenaires. Ce n'est pas un hasard si la traduction anglaise de ce traité connaît une seconde édition en 1588 (après celle de 1560), l'année de «l'Invincible Armada». En 1596, Sir Henry Knyvett publie The Defence of the Realme qui recommande un service militaire obligatoire pour tous les Anglais, idée reprise par Francis Bacon en 1608 dans un ouvrage au titre explicite : Of the True Greatness of the Kingdom Of Britain ${ }^{52}$.

L'hostilité à l'égard des étrangers prend de nouvelles proportions après le schisme. Nombre de visiteurs en parlent et on pourrait multiplier les exemples ${ }^{53}$. La xénophobie qu'éveille Philippe II d'Espagne lorsqu'il épouse Mary Tudor, ou Mary Stuart qui présente le triple handicap d'être catholique, écossaise et un peu française (par son mariage avec François Il) est célèbre. Moins connue est l'attitude des rebelles conservateurs du Pèlerinage de la Grâce (1536-37) : ils demandent que Mary Tudor, écartée du trône en 1534 retrouve son rang d'héritière en raison du danger d'une succession écossaise ${ }^{54}$ - les rivalités ancestrales avec le royaume du Nord prennent le pas sur l'orthodoxie catholique de Jacques $\mathrm{V}$ d'Ecosse. A la fin du siècle, le dramaturge Robert Greene donne une dimension morale (fréquente à l'époque) à l'animosité envers les Italiens :

[...] thou camest not alone, but accompanied with multitude of abhominable vices $[\ldots]$ infectious abuses and vaine glory, selfe loue, sodomie and strange poisonings, wherewith thou hast infected this glorious Iland $[\ldots]$ get thee home againe $[\ldots]$ and let mee [live] in my natiue home in England where I was bome and bred ${ }^{55}$.

Les Anglais sont d'autant plus disposés à se passer des étrangers qu'ils ont le sentiment de former une véritable nation. Ce sentiment n'est pas créé par le schisme mais la nouvelle organisation politico-religieuse vise à le renforcer. La loi sur les appels met les laïques et les clercs sur le même plan et les place, sans dif- 
férence aucune, sous la même autorité «pleine et entière» du souverain :

[...] unto whom a body politic, compact of all sorts and degrees of people divided in terms and by names of spiritualty and temporalty, be bounden and owe to bear next to God a natural and humble obedience; he being also institute and furnished by the goodness and sufferance of Almighty God with plenary, whole and entire power ${ }^{56}$.

Plus de double allégeance des ecclésiastiques, finis les «demisujets» et les «Anglais papistiqués» («papisticate»). L'idée d'unité nationale à l'intérieur du «corps politique» est précisée par Henry VIII lui même en 1543 lorsqu'il dit aux parlementaires :

[...] We as head and you as members are conjoined and knit together into one body politic 57 .

Comme les députés représentent le pays, c'est l'ensemble de la nation qui forme un seul corps.

On s'efforce de couler les nouvelles structures dans le vieux moule d'une conception organique de la société où chaque membre a son rôle à jouer, à une place déterminée. L'ordre divin qui prévaut dans le cosmos comme à l'intérieur du pays assigne une fonction («a vocation») à chacun. Le «corps politique» n'est pas un simple rassemblement d'individus mais un ensemble homogène où doivent prévaloir interdépendance et coopération ${ }^{58}$. Même si Luther avait insisté sur les droits de la conscience individuelle, Henry VIII et les auteurs du statut sur les appels ne considèrent que le tout. Comme au Moyen Age, l'individu n'existe pas mais le tout maintenant est la nation anglaise.

Un synonyme de «corps politique», le mot «commonwealth» connaît, entre 1535 et 1560 , une vogue exceptionnelle. Toute une littérature que nous qualifierions de sociale ou de socioéconomique étudie comment promouvoir le bien commun, comment éviter que l'intérêt personnel nuise à la communauté, au bien- être collectif ${ }^{59}$. Selon Thomas Starkey, celui qui recherche son plaisir ou sa tranquillité sans penser aux autres «cause un tort évident à son pays» ${ }^{60}$. Des auteurs comme Henry Brinklow et Robert Crowley souhaitent que le schisme soit l'occasion de réformes sociales et que les domaines confisqués aux monastères servent à créer des hôpitaux, des écoles ${ }^{61}$. Les responsabilités réciproques du gouvernement et des sujets - le premier apportant aide et protection, les seconds leur travail - définissent les conditions de l'harmonie générale. 
On insiste sur le fait que les mêmes personnes constituent à la fois l'Église et l'État ${ }^{62}$. Dès lors, le clergé ne saurait se prévaloir de privilèges ou d'immunités; les diverses franchises ou «libertés» ecclésiastiques sont supprimées (Ripon, Beverley, Durham, etc.), de même que le droit de sanctuaire ${ }^{63}$. Désormais, à quelques nuances près, la loi s'appliquera partout de la même façon. Les officialités perdent de leur influence, le droit canon recule au profit du droit coutumier. A la société bi-polaire d'avant le schisme succède un système centralisé (beaucoup plus qu'en France) et unifié.

Une réforme administrative de grande envergure renforce la puissance de l'État et l'unité du pays. Des «départements» (ancêtres des ministères) contrôlés par un Conseil privé réorganisé en 1536 restreignent le rôle peu rationnel de la Maison du roi et introduisent des méthodes de gouvernement beaucoup plus efficaces ${ }^{64}$. Le Conseil du Nord (à York) devient permanent après la révolte de 1536-37, le Conseil des Marches de Galles (à Ludlow) réduit les restes du système féodal dans cette région. Le Pays de Galles est plus fermement incorporé au royaume par des statuts de 1536 et $1543^{65}$. La perte de Calais en 1558 - qui achève de déconsidérer le régime de Mary Tudor - est tenue pour une catastrophe nationale mais elle ajoute à l'homogénéité de la nation et du territoire désormais «enchâssé dans la mer d'argent qui le protège comme un rempart ou comme une douve», pour reprendre le texte de Shakespeare. L'isolement géographique s'ajoute aux diverses réorganisations administratives, politiques et religieuses pour donner forme et cohésion au sentiment national.

Une communauté menacée d'invasion ne peut survivre que si elle fait taire en son sein toutes les forces centrifuges. Le besoin d'obéissance est élevé, après le schisme, au rang d'impératif catégorique à forte connotation religieuse.

Aux raisons traditionnelles - tout pouvoir vient de Dieu et l'épître aux Romains exige la soumission aux pouvoirs établis ${ }^{66}$ - s'en ajoutent d'autres liées à la position du roi à la tête de l'Eglise. Dans cette situation, le souverain est, pourrait-on dire, plus près de Dieu et l'obéissance est encore plus obligatoire. Des homélies sont publiées en 1547, puis une deuxième série en 1571 , qui doivent être lues en chaire par les ecclésiastiques semaine après semaine. Dans les deux cas, il y en a une sur l'obéissance qui condamne toute rébellion même contre un mauvais prince ${ }^{67}$. Un 
mauvais prince est d'ailleurs envoyé par Dieu pour punir les péchés de mauvais sujets.

De multiples serments - un engagement devant Dieu sont imposés à la population ou à certains groupes sociaux pour s'assurer qu'ils acceptent le nouveau régime ${ }^{68}$. Ceux qui refusent comme Thomas More, John Fisher et plusieurs Chartreux sont impitoyablement exécutés. Le problème - pour More en particulier - est de marquer les limites des institutions nationales : le parlement anglais n'avait pas autorité pour voter des textes contraires aux lois de la chrétienté :

Ce royaume n'étant qu'un membre et une petite part de l'Église, ne pouvait forger une loi particulière qui fût en désaccord avec la loi générale de l'Eglise Catholique Universelle du Christ, pas plus que la Cité de Londres, n'étant qu'un pauvre membre du royaume tout entier, ne pouvait faire une loi contre un acte du Parlement pour obliger tout le royaume ${ }^{69}$.

Inversement ceux qui se plient aux volontés officielles acceptent une totale autonomie législative du parlement et donc la nouvelle souveraineté de leur pays.

Certes de nombreuses mesures de coercition menacent les réfractaires. Entre 1534 et 1585, une dizaine de statuts précisent les cas de trahison soulignant l'importance croissante de l'idée de patrie puisque «trahison» ne définit pas que des atteintes à la personne ou au pouvoir du souverain, comme auparavant, mais tout ce qui peut prêter préjudice au régime établi.

Etant donné la situation intérieure et extérieure, chacun doit comprendre aussi que l'anarchie est le pire des dangers comme le répètent les homélies. Autrement dit, des raisons utilitaristes s'ajoutent aux considérations religieuses. C'est ce qu'exprime John Foxe, le martyrologiste, en 1576:

God has so placed us Englishmen here in one commonwealth, also in one church, as in one ship together: let us not mangle or divide the ship, which being divided perisheth... No storm so dangerous to a ship on the sea, as is discord and disorder in a weal public ${ }^{70}$.

La survie du pays est en jeu, toute division est suicidaire. Le schisme, souhaité par Dieu, selon Foxe, (cf. début de la citation), renforce la nécessité de l'obéissance.

Au total, les Anglais sont fiers, à la fin du XVI ${ }^{e}$ siècle, de l'ordre et de la discipline qui prévalent dans leur pays. En 1594, un 
opposant notoire, le député puritain Peter Wentworth, écrit, sans la moindre ironie, de la Tour de Londres où il est emprisonné :

Our nation is of itself more inclined to the subjection of our prince, to obedience to our laws to reason and to right than any other people which is between the two poles ${ }^{71}$.

Il faut remarquer que les rares auteurs qui envisagent la possibilité de ne pas obéir le font au nom de la loi de Dieu et de l'intérêt du pays. C'est ce que disent les catholiques jusqu'aux années 1580 quand ils prônent une intervention armée ${ }^{72}$; mais, sous une autre forme, ils le répètent quand, mis en échec par le patriotisme anglais, ils changent de politique et renoncent à la théorie des deux glaives. A propos de la succession d'Elizabeth, le jésuite Robert Parsons publie, sous un pseudonyme, un livre où il dit que c'est au peuple de choisir le prochain souverain. L'autorité du monarque est «a power delegate, or a power by Commission from the Commonwealth ${ }^{73}$. Cette conception «montante» du pouvoir implique une sorte de contrat entre gouvernant et gouvernés, grâce à la cérémonie du couronnement. Mais le corps, selon l'ancienne métaphore, a plus de pouvoir que la tête ${ }^{74}$, la nation est plus importante que celui qui la dirige.

La même idée a déjà été exprimée, en 1556, à l'autre extrémité de l'éventail politico-religieux. John Ponet (ou Poynet) qui avait été évêque (très réformiste) de Winchester sous Edward VI affirme que les souverains sont soumis à la loi de Dieu et aux lois positives de leur pays et que chacun doit plus de respect à sa patrie qu'à son prince :

Men ought to have more respect to their country, than to their prince : to the common wealth than to any one person. For the country and the common wealth is a degree above the king... And common wealths may stand well enough and flourish albeit there be no king $^{75}$.

Contrairement à ce qu'avait connu le Moyen Age, la forme «nationale» du patriotisme l'emporte ici sur la forme «monarchique» - une position très minoritaire au $\mathrm{XVI}^{e}$ siècle mais qui va se développer d'une façon considérable au XVIIe.

Si le schisme a pu apparaître d'abord comme une série de décisions politiques, si certains ont cru, en 1534, que seul le pouvoir juridictionnel du pape était aboli, ils se sont vite rendu compte que le climat religieux - sans parler de la doctrine - 
changeait beaucoup. Ce nouveau climat, une sorte d'exaltation permanente, a marqué de son empreinte le sentiment national, lui a donné sa force et sa coloration particulières.

A partir de l'idée (henricienne) que le prince retrouve les pouvoirs que Dieu avait confiés à ses prédécesseurs et délivre ses sujets de la tyrannie pontificale, on va passer à l'idée (protestante) que le pays retrouve la pureté du christianisme primitif. Un auteur anonyme écrit en 1571 :

The religion presently taught and professed in the Church at thys present, is no new reformation of thinges lately begonne, which were not before but rather a reduction of the Church to the pristine state of olde conformite ${ }^{76}$.

Dans les deux cas, Dieu a choisi l'Angleterre pour rectifier ce qui n'allait pas sur terre. En 1539, Richard Morison exhorte ses compatriotes à défendre les acquis du schisme en ces termes :

Why may we not think that the noble Henry VIII is the lion [...] sent by God to toss this wicked tyrant of Rome, to blow him out of all Christian regions? See you not to what honour God calls our nation? May we not rejoice that God has chosen our king, to work so noble a feat? let us fight this one field with English hands and English hearts ${ }^{77}$.

Comme après Azincourt, les Anglais chantent les louanges d'un Dieu qui a sélectionné leur nation entre toutes les autres, et qui est même (re)devenu anglais. A la naissance du futur Edward VI, Latimer se réjouit en ces termes :

[...] Thanks to our lord God, God of England! For verily he has shown himself God of England, or rather an English God... ${ }^{78}$

Beaucoup d'Anglais ont le sentiment, avant les puritains américains du XVII ${ }^{e}$ siècle, de revivre l'expérience du «peuple élu». John Foxe multiplie les parallèles avec le peuple d'Israël. Non seulement l'Angleterre a vaincu l'antéchrist mais elle est chargée d'accomplir les plus grands desseins de Dieu. C'est grâce à elle que la race humaine sera sauvée; le grand moment épique de l'histoire nationale s'inscrit dans une vision plus vaste encore et vraiment eschatologique.

A côté de Foxe dont l'ouvrage (Acts and Monuments) sera le plus lu en Angleterre après la Bible et façonnera donc l'esprit national, d'autres, avant Milton ${ }^{79}$ évoquent le même thème de la nation élue : John Stow (Annales... of England, 1580), Raphael 
Holinshed (Chronicles of England, Scotland and Ireland, 1577) ou John Lyly qui parle de la bonté de Dieu :

So tender a care hath He alwaies had of that England, as of a new Israel, his chosen and peculier people ${ }^{80}$.

La place d'Elizabeth, dans cette perspective, est claire : elle est la nouvelle Débora, prophétesse, juge et restauratrice d'Israel. Dieu lui-même est intervenu pour la protéger de ses ennemis (emprisonnement sous Mary Tudor) ${ }^{81}$ et une ballade intitulée «A Songe Betwene the Quenes Majestie and Englande» (1559) présente un dialogue entre la reine et «(her) dear lover England» où ce demier lui dit $« Y e$ are the handmaid of the lord ${ }^{82}$.

La protection divine se marque par la paix et la prospérité dont jouit l'Angleterre. En 1587, un député s'écrie au parlement

We that have lived in the eyes of all men so choked [...] with blessings of God beyond desert, we [...] confess that indeed the lord has vowed himself to be English ${ }^{83}$.

On comprend alors qu'il faille défendre un tel «paradis» comme dit Aylmer («England is the paradise and not Italy, as commonly they call it») ${ }^{84}$. A la veille de l'affrontement avec la flotte espagnole, Drake écrit à Elizabeth pour lui dire que les Anglais sont prêts à mourir pour leur patrie :

[...] we are all persuaded that God, the giver of all victories, will in mercy look upon your most excellent Majesty, and us your poor subjects, who for the defence of your Majesty, our religion and native country, have resolutely vowed the hazard of our lives ${ }^{85}$.

La victoire confirme l'aide de Dieu, la tempête qui achève de détruire l'Armada en est la preuve définitive.

Certes la reine est mentionnée en premier (avant la religion et la patrie) dans le texte de Drake mais d'une part c'est une lettre personnelle, d'autre part il y a plus ici que l'ancienne loyauté au monarque et on peut parler de fusion entre religion et patriotisme. Par ailleurs, comme le pensaient Ponet et Parsons, on peut changer le souverain, les deux autres sentiments restent, assez puissants pour inspirer l'ensemble de la nation (et pas quelques groupes sociaux), même si la personnalité d'Elizabeth émerveille ses sujets.

Il est donc clair que le sentiment national a changé de force et surtout de nature à la suite du schisme. Il est nettement teinté de 
sentiment religieux et comporte la nuance, puritaine, d'élection divine et de retour aux origines du christianisme. Il est aussi plus assuré, plus agressif et plus orgueilleux qu'avant 1534. John Lyly fait allusion à un «vieux dicton» selon lequel «all countries stande in neede of Britaine, and Britaine of none ${ }^{86}$.

\section{Conclusion}

Le schisme n'est évidemment pas la seule cause de l'essor du sentiment national. Le déclin du monde féodal, le besoin d'un pouvoir central fort après la guerre des Deux Roses, l'influence croissante des villes, des laïques et des couches sociales intermédiaires, les progrès du mercantilisme ont facilité ce développement. Leurs effets se seraient fait sentir sans le schisme comme on le remarque dans les pays qui n'ont pas rompu avec Rome. Mais ce phénomène a donné au patriotisme anglais une plus forte tonalité spirituelle qu'ailleurs.

On peut dire aussi, pour terminer, que l'influence conjuguée du schisme et du sentiment national transforme le système général de références intellectuelles, en particulier la conception médiévale d'un cosmos immuable, ordonné et hiérarchisé. Cette conception ne disparaît pas au XVIe siècle, c'est même dans Troilus and Cressida de Shakespeare qu'elle reçoit, en 1602, sa formulation la plus remarquable (discours d'Ulysse, acte I, scène 3 ). Il y a cependant incompatibilité au moins partielle entre cette vision statique et universelle et un sentiment national engendré par un État-nation qui revendique sa souveraineté, son identité propre, son droit à la différence.

On essaie, dans un premier temps, d'incorporer ces données nouvelles dans le système antérieur. L'homélie de 1547 sur l'obéissance qui, comme celle de 1571, a tant fait pour souder la nation anglaise, insiste sur l'ordre «naturel» et intangible que Dieu a mis dans la création ${ }^{87}$. Mais ce discours correspond de moins en moins à la réalité - peut-être le répète-t-on beaucoup pour se convaincre que la «mutabilité», que l'on craint tant, ne s'est pas encore installée. Les plus lucides ne sont pas dupes. Sir Thomas Smith, un des principaux penseurs politiques du XVIe siècle, sait bien qu'un régime - et donc les sentiments qu'il inspire - dépend des circonstances locales: 
[...] never in all pointes one common wealth doth agree with an other, no nor long time any one common wealth with it selfe. For al chaungeth continually ... ${ }^{88}$.

Les considérations sur le temps et le changement ne sont pas toutes pessimistes. «Ici et maintenant» valent bien «jadis et làbas» pour des auteurs comme Thomas Smith et surtout Francis Bacon qui se passent des idées médiévales ou humanistes sur des modèles lointains, sur un ailleurs jugé meilleur. Les élisabethains mettent de plus en plus l'accent sur leur propre temps, sur leur propre pays qui, pensent-ils, n'a jamais été plus florissant - qu'ils ont su rendre plus florissant que jamais ${ }^{89}$.

L'idée prend corps que c'est l'homme - et pas seulement Dieu et la «loi naturelle» - qui organise la vie en société, l'ordre politique. Les conceptions de Hobbes sur le caractère totalement artificiel de l'État ne sont pas encore de mise ${ }^{90}$ mais la transition commence lorsque s'accroissent les pouvoirs du parlement (c'est-àdire des hommes) à l'occasion du schisme, lorsque les Anglais élaborent un système politico-religieux dont ils sont fiers, lorsque cette fierté devient vraiment nationale.

Jean-Pierre MOREAU

Université de Limoges

\section{NOTES}

1. J. Lyly, «Euphues and His Eng1and» (1580), in The Complete Works of John Lyly, ed. R. Warwick Bond, Oxford, 1902, ed. 1967, vol. II, p. 197.

2. "The Britons doo excell such as dwell in the hoter countries», The Description of England in Shakespeare's Youth. Part II, ed. F.J. Furnivall, 1878, p. 153. «Our women whose beautie commonlie exceedeth the fairest of those of the maine...», ibid., p. 155. «Our women [...] after fortie begin to wrinkle apace, yet are they not commonlie so wretched and hard favoured to looke upon in their age, as the French women, and diverse of other countries», ibid. Il donne même dans le macabre lorsqu'il écrit «there hath not been seene in anie region so manie carcasses of the dead to remaine from time to time without corruption as in Britaine», ibid. 
3. J. Guy, Tudor England, Oxford University Press, 1988, p. 27. Cet auteur admet cependant qu'il existait «a keen sense of "Englishness" or "nationhood" in the fifteenth century», ibid.

4. J.W. Allen, A History of Political Thought in the Sixteenth Century, 1928 University Paperback, 1960, p. 125.

5. C'est ainsi que Henry VIII et ses partisans présentent les choses, notamment dans The Glass of Truth, 1532.

6. John W. McKenna, «How God became an Englishman», in Tudor Rule and Revolution, ed. D. J. Guth et J. W. McKenna, Cambridge, 1982, p. 28.

7. André Bossuat, «La formule "Le roi est empereur en son royaume". Son emploi au XVe siècle devant le Parlement de Paris», in Revue historique du droit français et étranger, $4^{\mathrm{e}}$ série, XXXIX, 1961, pp. 371-381.

8. W. Ullmann, «This Realm of England is an Empire», Journal of Ecclesiastical History, vol. 30, n 2, avril 1979, p. 177.

9. McKenna, art. cit., p. 34.

10. S. B. Chrimes, English Constitutional Ideas in the Fifteenth Century, Cambridge, 1936, pp. 172-73. L'idée que «The kingdom of the English is the kingdom of God» reprend aussi une tradition saxonne. Cf. J. W. McKenna, art. cit., p. 37.

11. Cité par McKenna, art. cit., p. 32. Ces paroles datent des années 1370-80.

12. Letters and Papers [...] of Henry VIII, ed. J. S. Brewer, 1862, vol. II (1), 1314, 353. (Désormais abrégé $L P$.)

13. C. Haigh (ed.), The Cambridge Historial Encyclopedia of Great Britain and Ireland, Cambridge University Press, 1985, p 94.

14. F. R. H. Du Boulay «The Fifteenth Century», in C. H. Lawrence (ed.), The English Church and the Papacy in the MiddleAges, Londres, Burns \& Oates, 1965, p. 211.

15. A. Birnie, An Economic History of the British Isles, 1935, University Paperback, 1969, p. 162.

16. W. S. Holdsworth, A History of English Law, Londres, Methuen, 1903-1946, vol. II, p. 472.

17. Idem, vol. IV, p. 327.

18. C. A. Sneyd (ed.), A relation ... of the Island of England ... about the Year 1500, Londres, 1847, pp. 20-21.

19. C. S. L. Davies, Peace, Print and Protestantism, 1450.1558, (The Paladin History of England), 1977, p. 17.

20. Texte dans G. Elton, The Tudor Constitution, Documents and Commentary, Cambridge, 1960, p. 357.

21. Ibid., p.356.

22. Texte: ibid., pp. $341-44$ et 349-51. 
23. «[...] Great and inestimable sums of money be daily conveyed out of this realm to the impoverishment of the same», ibid., p. 341.

24. An act for the exoneration of exactions paid to the see of Rome, ibid., pp. 351-55.

25. G. Marc'hadour, «Master More, the Common Lawyer», in H. Boventer (ed.), Thomas Morus Jahrbuch, 1991, Düsseldorf, 1991, p. 122.

26. Ibid.

27. G. Elton, Tudor Constitution, op. cit., p. 351.

28. Instruction du roi à ses envoyés à Rome (7 octobre 1530) in State Papers of King Henry VIII, 7261. (Dorénavant abrégé SP.)

29. Loi sur la succession (1534). Texte, Tudor Constitution, op. cit., p. 7.

30. Ibid. Voir aussi la loi sur les appels (1533): «to keep [the crown] from the annoyance as well of the see of Rome as from the authority of other foreign potentates», ibid., p. 345.

31. Ibid. p. 356.

32. Si Henry VIII reconnaît le titre d'évêque de Rome, certains comme Cranmer, archevêque de Canterbury, finiront par assimiler le pape à l'antéchrist - opinion courante sous Edward VI.

33. 25 Henry VIII, c.21. Texte, Tudor Constitution, op. cit., p. 354. Au début de 1534 , le roi lui-même avait présenté l'alternative dans une lettre à l'évêque de Durham: «Ye must chose eyther to folowe Criste and forsake the pope and his church or to folowe the pope and his churche and forsake Criste»SP. 6/9, fol. 225. p. 74 .

34. The Sermons of Bishop Latimer, Parker Society, 1844,

35. E. Hall, The Union of the two Noble ... Famelies of York and Lancaster, ed. Ellis, 1809, p. 788.

36. $S P .7,287$. En 1529, Simon Fish, pamphlétaire violemment anticlérical, avait attiré l'attention du roi sur le fait que les ecclésiastiques «exempt themselves from the obedience of your Grace», «A Supplication for the Beggars», 1529, in A. G. Dickens et D. Carr, The Reformation in England to the Accession of Elizabeth I, Documents of Modern History, E. Arnold, 1967, p. 17.

37. Les négociations avec Rome ont commencé en 1527. A tout moment un accord aurait pu être trouvé ; même après le mariage avec Anne Boleyn, les ponts ne sont pas coupés. Lorsque Paul III succède à Clément VII à la fin de 1534, alors que la rupture a eu lieu, le roi envoie un émissaire à Rome (Casale) et espère que le nouveau pape lui donnera raison.

38. Révélé et étudié par G. D. Nicholson en 1977 dans une thèse non publiée «The Nature and Function of Historical Argument in the Henrician Reformation» (Cambridge). 
39. Dépêches de l'ambassadeur impérial Chapuys : «Le Roy estoit Empereur et Pape absolu en son royaume» ( ${ }^{\text {er }}$ octobre 1530). Il «estoit avant les papes» et «avoit droit d'empire en son royaume sans recognoystre superieux» (13 janvier 1531).

40. Cf. plus haut, p. 78, n. 8.

41. J. Guy, «Thomas Cromwell and the Intellectual Origins of the Henrician Revolution», in A. Fox et J. Guy, Reassessing the Henrician Age, Humanism, Politics and Reform, 1500-50, Blackwell, 1986, pp. 158-59.

42. Ibid., pp. 160-61.

43. Dépêche de Chapuys, 13 janvier 1531. Selon le duc de Norfolk, un Anglais, autrefois, «avoit subiugué Rome, à scavoir Brennus». La légende de Constantin et de sa mère anglaise réapparaît tout au long du siècle. Cf. Spenser, The Faerie Queene, Livre II, chant $X$, strophes 59 et 60 .

44. Tudor Constitution, op. cit., p. 344.

45. J. Guy, op. cit., p. 162.

46. En 1521, au moment de la rédaction de son livre contre Luther, Henry aurait dit «we received from that see [Rome] our crown imperial», W. Roper, The Lyfe of Sir Thomas More, ed. E.V. Hitchcock, 1935, p. 68.

47. La deuxième loi sur les annates (1534) avait déjà interdit aux religieux d'assister aux assemblées générales de leur ordre sur le continent.

48. La peur d'une invasion est évoquée au parlement avant même la rupture avec Rome. Cf. dépêches de Chapuys du 21 février 1531 («grand danger de guerre») et du 2 mai 1532 (crainte de voir le royaume «destruyt et perdu»). Le premier de ces textes parle de «ce royaume qui est tributaire au siège apostolique».

49. J.-P. Moreau, Rome ou l'Angleterre, les réactions politiques des catholiques anglais au moment du schisme (1529-1553), Paris, P.U.F., 1984, pp. 260-261. Un sermon de Tunstall, l'évêque de Durham, mentionne Pole en ces termes «this pestilent man, worse than a pagan is not ashamed to destroy, if he could his natyve countrey», A Sermon of Cuthbert, Byshop of Duresme, Londres, Berthelet, 1539, F. 2vi.

50. Tunstall, ibid., F. 2 vii.

51. La cardinal Allen écrit en 1588, en prévision d'un succès de l'Armada, un texte où il traite Elizabeth de «the only poison calametie and destruction of our noble Churche and Cuntrie» (An Admonition to the Nobility and People of England ...). Ceci ne peut qu'horrifier et rassembler les Anglais bien qu'exprimant, aussi, une forme de patriotisme.

52. A. O. Meyer, England and the Catholic Church Under Queen Elizabeth, Londres, 1914, ed. 1967, pp. 345-346. 
53. Trois peuvent suffire: «The English are universally partial to novelty, hostile to foreigners...» $M$. Soriano, diplomate vénitien (1559) ; «They are [...] very suspicious, especially of foreigners, whom they despise», E. van Meteren, marchand flamand qui vivait à Londres sous Elizabeth; «[trades people] care little for foreigners, but scoff and laugh at them», compte rendu de la visite de Frédérick, duc de Würtemberg en Angleterre en 1592, in J. Hurstfield et A.G.R. Smith, Elizbethan People, State and Society, Documents of Modern History, Londres, Arnold, 1972, pp. 32-33-34.

54. «For the danger of the title that myght incurre to the crown of Scotland», Article 3 du «Conseil de Pontefract», LP., XI, 1246, p. 506.

55. Robert Greene, "A Quippe for an Upstart Courtier", 1592, in The Life and Complete Works [...] of Robert Greene, (12 vol.), The Huth Library, Vol. XI, I833, pp. 226-227.

56. Tudor Constitution, op. cit., p. 344.

57. Ibid., p. 270 (texte de R. Holinshed, Chronicles of England).

58. Cf. J.-P. Moreau, «Le "corps politique" aux XVI ${ }^{\mathrm{e}}$ et XVII siècles: avatars d'une métaphore», in M. T. Jones-Davies (ed.), Shakespeare et le corps, Paris, Belles Lettres, 1991, pp. 53-79.

59. W. R. D. Jones, The Tudor Commonwealth, 1529-1559, Londres, Athlone Press, 1970; G. R. Elton, Reform and Renewal, Thomas Cromwell and the Common Weal, Cambridge, 1973.

60. «dowth manyfest wrong to hys cuntrey», England in the Reign of Henry VIII : Dialogue (vers 1536) ed. J. M. Cowper, Londres, 1878, p. 2.

61. W. R. D. Jones, op. cit., p. 39.

62. S. Gardiner, De Vera Obedientia, 1535, in Obedience in Church and State, ed. P. Janelle, Cambridge, 1930, p. 94.

63. Voir la loi 27 Henry VllI, c. 24 de 1536 dans Tudor Constitution, op. cit., pp. 37-39.

64. Telle est la thèse défendue par G. R. Elton dans ses livres, notamment dans The Tudor Revolution in Government, Cambridge, 1953.

65. 27 Henry VIII, c. 26 et $34-35$ Henry VIII, c. 26 . L'Irlande est plus rétive et ne peut guère être considérée comme faisant partie du territoire anglais.

66. «Que chacun se soumette aux autorités. Car il n'y a point d'autorité qui ne vienne de Dieu», Rom. 13.1.

67. «An exhortation concerning Good Order and Obedience to Rulers and Magistrates» (1547); «An Homily against Disobedience and Wilful Rebellion» (1571). 
68. Serments sur la suprématie religieuse du roi, sur la succession au trône. Cf. J.-P. Moreau, Rome ou l'Angleterre?, op. cit., pp. 103-108,186-224.

69. W. Roper, «The Lyfe of Sir Thomas More», (v. 1560) ed. et trad. P. Leyris, Écrits de prison, 1953, p. 83.

70. J. Foxe, «Préface» à l'édition de 1576 de Acts and Monuments (The Book of Martyrs).

71. Cité par A. O. Meyer, England and the Catholic Church ..., op. cit., p. 328.

72. Cf. ci-dessus la citation du cardinal Allen, p. 89, n. 51.

73. R. Parsons (alias Doleman), A Conference about the Next Succession to the Crown of England (1594), ed. 1681, p. 59.

74. «The whole Body is of more Authority than the only Head», ibid., p. 31.

75. A Shorte Treatise of Politike Power, Strasbourg, 1556, ed,, fac-similé : W. S. Hudson, John Ponet, Advocate of Limited Monarchy, Chicago, 1942.

76. Cité par R. O'Day, The Debate on the English Reformation, Londres, Methuen, 1986, p. 7. On pourrait ajouter John Foxe qui parle de «the renewing of the old ancient church of Christ», ibid. p. 17 ou John Jewel (évêque de Salisbury de 1560 à sa mort en 1571): «We are come as near as we possibly could, to the church of the apostles and of the old catholic bishops and fathers», An Apology of the Church of England, 1562, ed. J. E. Booty, Ithaca, 1963, p. 121.

77. Cité par W. G. Zeeveld, Foundations of Tudor Policy, Harward, 1948, p. 233.

78. Cité par E.D. Marcu, op. cit., p. 78. Cf. aussi Matthew Parker archevêque de Canterbury: "Where Almighty God is so much English as he is, should not we requite his mercy?», ibid., p. 79. «The lord is on our side», écrit Drake à la veille de l'affrontement naval de 1588 (cité par A.O. Meyer, op. cit., p. 314) comme l'avait dit Tunstall en 1539 (cf. plus haut).

79. Milton (qui, à la différence de Jewel - cf. ci-dessus, n. 76. - trouve la réforme incomplète) écrit dans Areopagitica (1644) : «God is decreeing to begin some new and great period in His Church, even to the reforming of Reformation itself : what does He then but reveal Himself to His servants, and as His manner is, first to His Englishmen», Milton's Prose Writings, ed. K. M. Burton, (Everyman), 1958, p. 177.

80. Euphues and His England op. cit., p. 205.

81. John Aylmer (évêque de Londres de 1577 à 1594), An Harborowe for Faithful and True Subjects, 1559.

82. Texte, R. O'Day, The Debate on the English Reformation, op. cit., p. 21. Ce texte insiste sur les épreuves subies par Elizabeth et 
sur la protection de Dieu : «But the lorde of his might / defend the in right».

83. Cité par Marcu, op. cit., p. 80.

84. Ibid., John of Gaunt, dans Richard Il, parle de «This other Eden, demi-paradise» (Acte Il, sc. 1, v. 42).

85. Cité par A. O. Meyer, op. cit., p. 315. Au même moment, quand Elizabeth passe ses navires en revue, une chanson lui est dédiée qui exprime le même sentiment :

Where many [a captain] did say and swear

To live and die for England

And would not ask a penny pay...

But of their own would find a stay

To serve her Grace for England.

86. Euphues and his England op. cit., p. 196.

87. «Almighty God hath created and appointed all things in heaven, earth and waters in a most excellent and perfect order», Extraits dans Tudor Constitution, op. cit., p. 15.

88. Sir Thomas Smith, De Republica Anglorum, 1565, ed. Alston, 1906, p. 33.

89. S. L. Collins, From Divine Cosmos to Sovereign State, Oxford, 1989, passim.

90. «For by Art is created that great Leviathan called a Commonwealth, or State (in latin Civitas) which is but an Artificiall Man», Leviathan, «Introduction» (ed. Everyman, 1973, p.1). 